Chapter 9

\title{
Association Between HLA Gene Polymorphism and Antiepileptic Drugs-Induced Cutaneous Adverse Reactions
}

Yuying Sun and Yongzhi Xi

Additional information is available at the end of the chapter

http://dx.doi.org/10.5772/57513

\section{Introduction}

Epilepsy is a chronic, recurrent, and transient brain dysfunction syndrome caused by recurrent seizures due to abnormal firing of brain neurons, and it is one of the most common neurological disorders. The incidence of epilepsy is associated with age; the highest prevalence is generally thought to be under 1 year of age, followed by a gradual reduction after1-10 years. In China, the male to female ratio of epilepsy is between 1.15:1 and1.7:1, and no significant differences associated with race have been found.

The main treatment for epilepsy is medication, with antiepileptic drugs (AEDs) as the principal method. After systemic long-term treatment, most epilepsy patients can be cured by medication. Because new AEDs have come into the market, adverse effects have been significantly reduced, and thus, AEDs treatments have become more acceptable to most epilepsy patients. Among the AEDs, aromatic antiepileptic drugs (AAEDs) are the most commonly used. This class of drugs was named for their similar chemical structures and their possession of benzene rings. Currently, commonly used AAEDs in the clinicinclude carbamazepine (CBZ), oxcarbazepine (OXC), phenobarbital (PB), lamotrigine (LTG), and phenytoin (PHT), mainly used in treatments foridiopathic generalized epilepsy with good efficacy. However, adverse reactions such as rash, fever, and organ damagecan occur. The most common reaction is cutaneous adverse drug reactions (CADRs), which includes mild maculopapular eruptions (MPE);drug hypersensitivity syndrome (HSS), as well as life-threatening reactions such as severe cutaneous reactions (SCRs)(Stevens-Johnson syndrome[SJS] and toxic epidermal necrolysis [TEN]), the mortality rate of which is as high as $40 \%$, resulting in serious socio-economic and family burdens. 
In individuals taking AEDs, the overall MPE incidence rate is $2.8 \%$; incidence rates of rash caused by PHT, LG, and CBZ are higher at 5.9\%, 4.7\%, and 3.7\%, respectively. In comparison, the incidence rates of SCRs(including HHS, SJS, and TEN) caused by the above drugs are lower. A population survey in Germany indicated that in patients who had just began AEDs treatment, the incidence rate of CBZ-SJS/TEN was 1.4/10,000, whereas the incidence rates of LTGSJS/TEN, PB-SJS/TEN, and PHT-SJS/TEN were 2.5/10,000, 8.1/10,000, and 8.3/10,000, respectively. SJS and TEN are considered forms of the same disease at different stages, manifesting as blister-like rashes with skin peeling, and affecting the skin, mucous membranes, organs, visceral trunk, and limbs. SJS is characterized by total area of skin detachment of less than $10 \%$, whereas TEN is defined by an area of detachment greater than $40 \%$, and rates in between are considered SJS/TEN overlap. TEN is more severe, with mortality as high as $40 \%$. Although the incidence rates of SJS and TEN are low, mortality rates nevertheless reach 10 $50 \%$. Thus, avoiding SJS/TEN is one of the major challenges during AEDs treatment. Recent studies have indicated that SCRs such as SJS and TEN induced by AAEDs are associated with the $H L A-B^{*} 1502$ allele.

\section{Current cADRs prevalence in various countries and regions}

In-depth genetics studies on AEDs-induced adverse reactions suggest that incidence rates of AEDs-induced SJS and TEN vary among ethnic groups and that the associations with relevant loci are different. In Europe, CBZ is the most common drug causing cADRs, with an incidence rate of $8.2 \%$, followed by $\mathrm{PB}$ at $5.3 \%$, and $\mathrm{PHT}$ at $5.0 \%$. The prevalence rates of cADRs induced by CBZ are different depending on the country and/or region, at 5.88\% in Australia, $6.60 \%$ in Japan, $27.70 \%$ in Singapore, $35.70 \%$ in Malaysia, $19.00 \%$ in India, and $26.00 \%$ in Taiwan. The prevalence rates of cADRs induced by PHT are also largely variable among different countries and regions, at 5.00\% in Europe, 5.88\% in Australia, 14.20\% in Malaysia, 19.00\% in India, and $4.30 \%$ in Taiwan. A similar phenomenon has also been observed in the prevalence of cADRs induced by other drugs in different countries and regions. CBZ is the main SJS/TEN-inducing drug in Southeast Asian countries and regions. The incidence rate of CBZ-SJS/TEN in Taiwan is approximately 59/10,000 each year, 41/10, 000 in Malaysia, and 55/10,000 in the Philippines, while it is relatively low in the United States and Europe, at 2/10,000 in the U.S.A., 9/10,000 in the United Kingdom, and 5/10,000 in France.

\section{Association between AEDs-induced cutaneous adverse reactions and HLA alleles}

Detailed studies in human genomics and pharmacogenomics have demonstrated a relationship between drug-induced cADRs and human leucocyte antigen (HLA) genes. HLA genes are located on human chromosome 6 p21.3, and are a group of closely linked multiple alleles that include more than 100 loci and a total of 554 alleles, spanning 3,600 k band representing $1 / 3,000$ of the entire human genome. It is a major gene system that regulates human-specific 
immune responses and individual differences in disease susceptibility, with apparent specificity in different ethnicities or populations of the same ethnicity.HLA genes can be divided into three classes: HLA class I, class II, and class III, on the basis of the structural expression, tissue distribution, and functions of the encoded proteins. HLA-I genes include A, B, and C loci; HLA-II genes consist of DR, DQ, and DP subregions; and HLA-III genes reside between HLA-I and HLA-II genes, and are mainly related to the complement system. Disease-related studies have generally been focused on HLA-I and HLA-II genes.

\subsection{Correlation between cADRs incidence and the $H L A-B * 1502$ allele in different countries and regions}

Recent studies have shown that the incidence of CBZ-SJS/TEN is strongly associated with HLA$B^{*} 1502$ in Han Chinese, while its frequency is low in Japanese and Caucasians and not associated with the $H L A-B^{*} 1502$ allele. Chung et al.in Taiwan first reported the association between the $H L A-B^{*} 1502$ allele and CBZ-SJS/TEN. In 2004, they published a study in Nature on HLA gene polymorphisms in 44 cases of CBZ-SJS/TEN in Han Chinese living in Taiwan, which also included 101 CBZ-tolerant patients and 93 cases with a positive CBZ history as study controls. The results indicated that all CBZ-SJS/TEN cases were positive for HLA$B^{*} 1502(100 \%)$, whereas only $3 \%$ and $9 \%$ were positive, respectively, in the other two groups. This finding revolutionized the research field on cADRs and AEDs, and prompted further studies on the $H L A-B^{*} 1502$ allele. A study in Thailand revealed that among 42 cases of SJS/TEN caused by CBZ, 37 were positive for $H L A-B^{*} 1502$, which implies that the HLA$B^{*} 1502$ allele is a high-risk factor for CBZ-SJS/TEN occurrence. In the Han Chinese population, $H L A-B^{*} 1502$ genotyping in CBZ-SJS/TEN patients showed $100 \%$ sensitivity and $97 \%$ specificity. Avoiding oral CBZ in $H L A-B^{*} 1502$-positive patients decreased SJS/TEN incidence, while $H L A-B^{*} 1502$-negative patients rarely show adverse reactions and the risk of CBZ-SJS/TEN is low. Thus, $H L A-B^{*} 1502$ genetic screening in the clinic is important for the use of AEDs. Some experts have suggested that in Asian populations, $H L A-B^{*} 1502$ genetic screening should be performed before taking CBZ and related AAEDs, and CBZ should be avoided in those positive for $H L A-B^{*} 1502$; valproic acid, levetiracetam, or topiramate should be used instead as alternative treatments.

A study by Hung et al. on the Han Chinese population found that the HLA-B*1502-positive rate is as high as $100 \%(3 / 3)$ in OXC-induced SCR (AXC-SJS) cases. Locharenkul et al. reported four cases of SCR induced by PHT (PHT-SJS) in Thailand who were all positive for HLA$B^{*} 1502$, which further confirmed the association between SJS/TEN and the HLA-B*1502 allele in Southeast Asian countries. The frequency of the $H L A-B^{*} 1502$ allele shows significant regional variations: $7.1 \%$ in South China, $1.9 \%$ in North China, $4.3 \%$ in Taiwan, $7.2 \%$ in Hong Kong, $6.1 \%$ in Thailand, $8.4 \%$ in Malaysia, $0.1 \%$ in Japan, $0.4 \%$ in South Korea, and lower or almost zero in European countries such as Germany and France. The results in European populations were also different from those in Southeast Asian countries. One study in Europe revealed that among the 12 cases of CBZ-SJS/TEN, 8 were Caucasian and did not carry the $H L A-B^{*} 1502$ allele, whereas the other 4 were positive for $H L A-B^{*} 1502$ and of Asian origin (China, Vietnam, and Cambodia). Thus, the frequency of $H L A-B^{*} 1502$ is low in Japan and in 
European Caucasians, and consequently, the incidence of CBZ-SJS/TEN is low. Conversely, the frequency of $H L A-B^{*} 1502$ is relatively high in Southeast Asian countries and regions (Taiwan, Hong Kong, Malaysia, Singapore, Thailand, and others)and the incidence of CBZ$\mathrm{SJS} / \mathrm{TEN}$ is also high, which further proves the strong association between CBZ-SJS/TEN incidence and $H L A-B^{*} 1502$. Therefore, the U.S.A FDA has recommended that in Han Chinese and Southeast Asian populations, $H L A-B^{*} 1502$ screening should be performed before prescribing $C B Z$, and individuals who are positive for $H L A-B^{*} 1502$ should be cautious when taking CBZ in order to reduce CBZ-SJS/TEN incidence.

\subsubsection{Relationship between the HLA-B*1502 allele and SJS/TEN due to other AEDs}

Due to the structural similarity and clinical cross-reactivity of AAEDs, several subsequent studies have been conducted regarding the correlation between other AAEDs and the HLA$B^{*} 1502$ allele. In studies conducted in Hong Kong, Taiwan, and Mainland China, no association was found between $H L A-B^{*} 1502$ and LTG-SJS/TEN in the Han Chinese population, a weak association was seen with PHT-SJS/TEN relative to CBZ-SJS/TEN, and all three OXC-SJS cases were positive for $H L A-B^{*} 1502$. However, due to the small number of cases, the correlation between SJS/TEN caused by other AAEDs and HLA-B*1502 remains to be determined using a larger sample size.

\subsubsection{Mechanism of the association between HLA-B*1502-positive patients and AAEDs-SJS/TEN}

Currently, the mechanism of severe cutaneous adverse reactions (SCAR) induced by AAEDs is unclear. Most scientists believe that provocation of the media results in severe symptoms within 2-3 days. In addition, a large number of infiltrating inflammatory cells are found in patient lesions, and increased dosage of CBZ significantly shortened the time for inflammatory cells to appear, which aggravated the symptoms. Therefore, the mechanistic origin likely lies in activation of the immune system. Cytotoxic T lymphocytes cause skin lesions in SJS/TEN patients with a common indicator of keratinocyte apoptosis induced by cytotoxic T-cells, and T-cells in the blister fluid of patients are mainlyCD8+ T-cells, implicating CD8+ T-cell-mediated cytotoxicity. Drugs such as CBZ and its metabolites are small chemicals, insufficient to induce immune responses, and thus the hapten hypothesis was proposed; that a specific drug or its metabolite covalently interacts with a protein or a polypeptide as a hapten, and is processed by cells and presented to the MHC molecules, resulting in HLA-specific T-cell activation. Another hypothesis is the p-i concept(direct pharmacological interaction between drug and immune receptor), i.e., the drug can be directly and non-covalently associated withT-cell receptors that match MHC molecules. Both hypotheses indicate that skin adverse reactions are triggered through interactions with specific MHC molecules, T-cell receptors, and drug-modified antigens. In 2007, Yang et al. studied HLA-B ${ }^{*} 1502$-bound peptides and found that CBZ or its metabolites non-covalently interacted with HLA-B ${ }^{*} 1502$-bound peptides, which resulted in cytotoxic T-cell-mediated apoptosis in SJS/TEN patients. This result favored the $\mathrm{p}-\mathrm{i}$ concept. However, current studies have not been able to explain the mechanism of SJS/TEN incidence in HLA$B^{*} 1502$-negative patients, which requires further investigation. 


\subsubsection{Correlation of $C A D R$ soccurrence and other HLA loci}

HLA-B* 1502 is associated with CBZ-SJS/TEN and is also polymorphic among ethnic groups. Japanese scientists performed a study on 15 CBZ-induced cADRs patients (10 MPE and 5 SJS) and found that the $H L A-B^{*} 1518, H L A-B^{*} 5901$, and $H L A-C^{*} 0704$ alleles were highly significantly associated with SCR risk, and that the haplotype HLA- $A^{*} 2402-B^{*} 5901-C^{*} 0102$ was significantly associated with SCR risk. This study revealed that individuals with these alleles may have an increased CBZ-cADRs incidence rate and that that HLA-B ${ }^{*} 5901$ locus is a risk locus for CBZSJS in the Japanese population. Another study in Japan indicated that $H L A-B^{*} 5801$ locus positivity was significantly associated with CBZ-SJS. A study in Europe also discovered an association with $H L A-A^{*} 3101$, with a frequency of approximately $2-5 \%$ in Northern Europe and that this gene locus was significantly associated with HSS $(P=3.5 \times 10-8)$ and MPE $(P=$ $1.1 \times 10-6)$, and a risk factor for HSS, MPE, and SJS/TEN. People of Northern European descent carrying the HLA- $A^{*} 3101$ allele had an increased CBZ-HHS incidence (from $5.0 \%$ to $26.0 \%$ ); conversely, those lacking this allele had a decreased incidence rate (from $5 \%$ to $3.8 \%$ ). A recent report showed that several cases of CBZ-SJS/TEN in HLA-B*1502-negative children in Han Chinese were found to be associated with $H L A-A^{*} 2402$.Therefore, $H L A-B^{*} 1502$-negative patients should also be closely observed after taking CBZ.

Scientists in Taiwan have also found that $H L A-B^{*} 1301, C w^{*} 0801$, and DRB1*1602 were associated with PHT-SJS/TEN. A study in Europe indicated that in addition to the HLA-B locus, $H L A-C w^{*} 0718, D Q B 1^{*} 0609, A^{*} 6801$, and $D Q B 1^{*} 1301$ were borderline-associated with LTG-SJS/ TEN, and HLA-B*5801 and $B^{*} 38$ were weakly associated with LTG-SJS/TEN. However, the sample sizes in these studies were small; thus, further investigations are necessary to determine if the above genes are risk factors for LTG-SJS/TEN or PHT-SJS/TEN.

\section{Summary}

In summary, cADRs incidence resulting from AEDs varies among different regions, and the associations with related gene loci are not consistent. $H L A-B^{*} 1502$-positivity is more frequent in Han Chinese and Southeast Asian populations than in populations from Japan and European countries, and is strongly associated with CBZ-SJS/TEN. HLA-B*1502 screening is important in choosing to use AEDs in the clinic; however, close observation is equally necessary for $H L A-B^{*} 1502$-negative patients to avoid CBZ-SJS/TEN. Because of the high mortality rate of SJS/TEN, HLA-B*1502-positive patients should avoid using CBZ, and instead chose to use levetiracetam, sodium valproate, topiramate, or other non-AAEDs. In individuals of Northern European ancestry, CBZ-SJS/TEN incidence is not associated with HLA-B*1502, but it is associated with $H L A-A^{*} 3101$, carriers of which have a significantly increased risk of cADRs. In Japan, HLA-B*5901 and HLA-B*5801 loci are risk factors for CBZ-SJS/TEN. Further studies will likely discover more AEDs-cADRs-associated gene loci, which will enrich the field of pharmacogenetics to provide more evidence for the clinical use of AEDs, reduce the incidence of cADRs, improve AEDs efficacy, and significantly reduce the risk of adverse drug reactions such as cADRs. 


\section{Acknowledgements}

Supported by grants from the State Key Development Program for Basic Research of China (No.2003CB515509 and 2009CB522401) and from National Natural Scientific Foundation of China(No.81070450 and 30470751) to Dr. X.-Y.Z.

\section{Author details}

Yuying Sun and Yongzhi $\mathrm{Xi}^{*}$

*Address all correspondence to: xiyz@yahoo.com

Department of Immunology and National Center for Biomedicine Analysis, Beijing Hospital Affiliated to Academy of Medical Sciences, Beijing, PRC

\section{References}

[1] Ling ZY, Sun Y, Jiang JF. New development about safe questions of carbamazepine in epileptic. China Mod Med, 2010, 17(13): 13-14.

[2] Beswick TC, Cohen JB. Dose-related levetiracetam-induced reticulated drug eruption. J Drugs Dermatol, 2010, 9(4): 409-410.

[3] Ouyan H, Liu GG. A review over adverse drug reaction of anti-epileptic drugs. J Pediatr Pharm, 2005, 11(4): 57-59.

[4] Lonjou C, Borot N, Sekula P, et al. A European study of HLA-B in Stevens-Johnson syndrome and toxic epidermal necrolysis related to five high-risk drugs. Pharmacogenet Genomics, 2008, 18(2): 99-107.

[5] Gao MM, Shi YW, Yu MJ, et al. Association between cutaneous adverse reactions to antiepileptic drugs and HLA-B* 1502 allele. Chin J Neuromed, 2009, 8(5): 493-496.

[6] Horton R, Wilming L, Rand V, et al. Gene map of the extended human MHC. Nat Rev Genet. 2004, 5(12): 889-899.

[7] Chung WH, Hung SI, Hong HS, et al. Medical genetics: a marker for Stevens-Johnson syndrome. Nature. 2004, 428(6982):486.

[8] Hung SI, Chung WH, Jee SH, et al. Genetic susceptibility to carbamazepine-induced cutaneous adverse drug reactions. Pharmacogenet Genomics. 2006, 16(4): 297-306. 
[9] Man CB, Kwan P, Baum L, et al. Association between HLA-B*1502 allele and antiepileptic drug-induced cutaneous reactions in Han Chinese. Epilepsia. 2007, 48(5): 1015-8.

[10] Wang GQ, Zhou YQ, Zhou LM, et al. Association between HLA-B*1502 Allele and carbamazepine-induced cutaneous adverse reactions in han people $\mathrm{f}$ China mainland. J Sun Yat sen Univ Med Sci, 2010, 31(6):828-832.

[11] Mehta TY, Prajapati LM, Mittal B, et al. Association of HLA-B*1502 allele and carbamazepine-induced Stevens-Johnson syndrome among Indians.Indian J Dermatol Venereol Leprol. 2009, 75(6): 579-582.

[12] Ding WY, Lee CK, Choon SE. Cutaneous adverse drug reactions seen in a tertiary hospital in Johor, Malaysia. Int J Dermatol. 2010, 49(7):834-841.

[13] Locharernkul C, Loplumlert J Limotai C, et al. Carbamazepine and phenytoin induced Stevens-Johnson syndrome is associated with HLA-B*1502 allele in Thai population. Epilepsia. 2008, 49(12):2087-2091.

[14] Lonjou C, Thomas L, Borot N, et al. A marker for Stevens-Johnson syndrome ...: ethnicity matters. Pharmacogenomics J. 2006, 6(4):265-268.

[15] Kaniwa N, Saito Y, Aihara M, et al. HLA-B locus in Japanese patients with anti-epileptics and allopurinol-related Stevens-Johnson syndrome andtoxic epidermal necrolysis. Pharmacogenomics. 2008, 9(11) :1617-1622.

[16] Kaniwa N, Hasegawa R. Exploratory studies on genetic biomarkers related to serious drug adverse reactions. Kokuritsu Iyakuhin Shokuhin Eisei Kenkyusho Hokoku. 2009, (127):1-14.

[17] Ikeda H, Takahashi Y, Yamazaki E, et al. HLA class I markers in Japanese patients with carbama- zepine-induced cutaneous adverse reactions. Epilepsia. 2010, 51(2): 297-300.

[18] Hung SI, Chung WH, Liu ZS, et al. Common risk allele in aromatic antiepilepticdrug induced Stevens-Johnson syndrome and toxic epidermal necrolysis in Han Chinese. Pharmacogenomics, 2010, 11(3): 349-356.

[19] Kazeem GR, Cox C, Aponte J, et al. High-resolution HLA genotyping and severe cutaneous adverse reactions in lamotrigine treated patients. Pharmacogenet Genomics, 2009, 19(9): 661-665.

[20] Lin LC, Lai PC, Yang SF, et al. Oxcarbazepine-induced Stevens-Johnson syndrome: a case report. Kaohsiung J Med Sci, 2009, 25(2): 82-86.

[21] Kuwbara S. Guillain-Barré syndrome: epidemiology, pathophysiology and management. Drugs, 2004, 64(6):597-610. 
[22] Nassif A, Bensussan A, Borothee G, et al. Drugs pecific cytotoxic T-cells in the skin lesions of a patient with toxic epidermal necrolysis. J Invest Dermatol, 2002, 118(4): 728-733.

[23] Nassif A, Bensussan A, Boumsell L, et al. Toxic epidermal necrolysis: effector cells are drug-specific cytotoxic T cells. J Allergy Clin Immunol. 2004, 114(5):1209-1215.

[24] Pichler WJ. Pharmacological interactionof drugs with antigen specific immunereceptors: the pi concept. Curr Opin Allergy Clin Immunol, 2002, 2(4): 301-305.

[25] Wu Y, Sanderson JP, Farrell J, et al. Activation of T cells by carbamazepine and carbamazepine metabolites. J Allergy Clin Immunol. 2006, 118(1): 233-241.

[26] Wu Y, Farrell J, Pirmohamed M, et al. Generation and characterization of antigenspecific CD4+, CD8+, and CD4+CD8+ T-cell clones from patients with carbamazepine hypersensitivity. J Allergy Clin Immunol. 2007, 119(4):973-981.

[27] Yang CW,Hung SI,Juo CG, et al. HLA-B*1502-bound peptides:implications for the pathogenesis of carbamazepine-induced Stevens-Johnsonsyndrome. J Allergy Clin Immunol. 2007, 120(4):870-877.

[28] Chung WH, Hung SI, Chen YT. Genetic predisposition of life-threatening antiepileptic-induced skin reactions. Expert Opin Drug Saf, 2010, 9( 1) :15-21.

[29] Toledano R, Gil-Nagel A. Adverse effects of antiepileptic drugs. Semin Neurol, 2008, 28(3):317-327.

[30] Arif H, Buchsbaum R, Weintraub D, et al. Comparison and predictors of rash associated with 15 antiepileptic drugs. Neurology, 2007, 68(20): 1701-1709.

[31] Mockenhaupt M, Messenheimer J, Tennis P, et al. Risk of Stevens-Johnson syndrome and toxic epidermal necrolysis in new users of antiepileptics. Neurology, 2005, 64(7): 1134-1138.

[32] Mockenhaupt M, Viboud C, Dunant A, et al. Stevens-Johnson syndrome and toxic epidermal necrolysis assessment of medication risks with emphasis on recently marketed drugs. The EuroSCAR-study. J Invest Dermatol, 2008 128(1): 35-44.

[33] Tassaneeyakul W, Tiamkao S, Jantararoungtong T, et al. Association between HLA$\mathrm{B}^{*} 1502$ and carbamazepine-induced severe cutaneous adverse drug reactions in a Thai population. Epilepsia, 2010, 51(5): 926-930.

[34] Chang CC, Too CL, Murad S, et al. Association of HLA-B*1502 allele with carbamazepine-induced toxic epidermal necrolysis and Stevens-Johnson syndrome in the multi-ethnic Malaysian population. Int J Dermatol, 2011, 50(2): 221-224.

[35] Shi YW, Min FL, Liu XR, et al. Hla-B alleles and lamotrigine-induced cutaneous adverse drug reactions in the Han Chinese population. Basic Clin Pharmacol Toxicol, 2011, 109, 1: 42-46. 
[36] Min FL, Shi YW, Liu XR, et al. HLA-B*1502 genotyping in two Chinese patients with phenytoin-induced Stevens-Johnson syndrome. Epilepsy Behav, 2011, 20(2): 390-391.

[37] Alfirevic A, Jorgensen AL, Williamson PR, et al. HLA-B locus in Caucasian patients with carbamazepine hypersensitivity. Pharmacogenomics, 2006, 7(6): 813-818.

[38] Ozeki T, Mushiroda T, Yowang A, et al. Genome-wide association study identifies HLA-A ${ }^{*} 3101$ allele as a genetic risk factor for carbamazepine-induced cutaneous adverse drug reactions in Japanese population. Hum Mol Genet, 2011, 20(5): 1034-1041.

[39] Liao WP, Shi YW, Min FL. HLA-B*1502 screening and toxic effects of carbamazepine. N Engl J Med, 2011, 365(7): 672-673.

[40] Shi YW, Min FL, Qin B, et al. Association between HLA and Stevens-Johnson syndrome induced by Carbamazepine in Southern Han Chinese genetic markers besides B¹502. Basic Clin Pharmacol Toxicol. 2012, 111(1):58-64. 7843.

[41] Hung SI, Chung WH, Jee SH, et al. Genetic susceptibility to carbamazepine-induced cutaneous adverse drug reactions. Pharmacogenet Genomics, 2006, 16(4): 297-306.

[42] An DM, Wu XT, Hu FY, et al. Association study of lamotrigine-induced cutaneous adverse reactions and HLA-B*1502 in a Han Chinese population. Epilepsy Res, 2010, 92(2-3): 226-230.

[43] Hu FY, Wu XT, An DM, et al. Pilot association study of oxcarbazepine-induced mild cutaneous adverse reactions with HLA-B*1502 allele in Chinese Han population. Seizure, 2011, 20(2): 160-162.

[44] Chen P, Lin JJ, Lu CS, et al. Carbamazepine-induced toxic effects and HLA-B 1502 screening in Taiwan. N Engl J Med, 2011, 364(12): 1126-1133. 
